\title{
Selbstmordverhinderung im freiheitlichen Staat
}

Bei der Frage nach dem grundrechtlichen Schutz des Selbstmordes handelt es sich gleichsam um die Gretchenfrage der Grundrechtsdogmatik: Reicht die Freiheit des Einzelnen so weit, dass er sich - um es mit dem Bundesverfassungsgericht zu sagendie »vitale Basis«" seiner Freiheit selbst entziehen darf? Die Wissenschaft hat die Problematik bislang noch keiner konsensfähigen Lösung zugeführt, was auch deshalb nicht so dringend erscheinen mag, weil die Relevanz des Problems aus Sicht der Rechtspraxis begrenzt sein dürfte. Dass es sich bei der Frage allerdings nicht nur um akademische Gedankenspiele handelt, zeigt beispielsweise die weiterhin schwelende Debatte zur Legitimität von Sterbehilfe, die ohne eine dogmatisch saubere Grundlegung zu der Frage des Verfügungsrechts des Einzelnen über sein Leben nicht befriedigend geführt werden kann. Daher wendet sich der vorliegende Beitrag dieser Grundfrage noch einmal zu. Er kritisiert die bisherigen Lösungsansätze als unbefriedigend und teilweise widersprüchlich und macht einen eigenen Vorschlag, mit dem die Rettung von Selbstmördern im freiheitlichen Staat ohne systematische Brüche in vielen Fällen gerechtfertigt werden kann.

\section{Das Recht auf Selbstmord}

\section{Schutz des Selbstmordes durch das allgemeine Freiheitsrecht}

Versteht man Art. 2 I GG als allgemeines Freiheitsrecht, so liegt es nahe, auch den Selbstmord als - letzte - Freiheitsbetätigung geschützt zu sehen. ${ }^{2}$ Dies ist allerdings nicht unumstritten. Ein Teil der Literatur hält der weiten Auslegung des Art. 2 I GG drei Einwände entgegen.

Der erste scheint zunächst so evident zu sein, dass zumeist auf eine eingehende Begründung verzichtet wird: Art. 2 I GG gestatte nur die »Entfaltung « der Persönlichkeit, nicht aber deren Zerstörung. ${ }^{3}$ Bei einer reinen Wortlautbetrachtung mag dieses Argument zutreffend erscheinen. Erstaunlicherweise diskutieren die Autoren, die die-

1 BVerfGE 39, 1 (42).

2 So Deger, Waffeneinsatz gegen Selbstmörder?, in: NVwZ 2001, 1229 (1230); Hufen, In dubio pro dignitate, in: NJW 2001, 849 (851); Jarass, in: Jarass/Pieroth, GG, 7. A. 2004, Art. 2, Rn. 8; Kunig, in: von Münch/Kunig (Hrsg.), GG, 5. A. 2000, Art. 2, Rn. 12; Murswiek, in: Sachs (Hrsg.), GG, 3. A. 2003, Art. 2, Rn. 211; Schulze-Fielitz, in: Dreier (Hrsg.), GG, 2. A. 2004, Art. 2 II, Rn. 32; Sachs, Grundrechte, 2000, B 2, Rn. 81; Wagner, Selbstmord und Selbstmordverhinderung, 1975, S. 90 ff.; Wassermann, Das Recht auf den eigenen Tod, in: DRiZ 1986, 291 (293).

3 Frotscher, Der Schutz der Allgemeinheit und der individuellen Recht im Polizei- und Ordnungsrecht, in: DVBl. 1976, 695 (702); Götz, Allgemeines Polizei- und Ordnungsrecht, 13. A. 2001, Rn. 109; Lorenz, Recht auf Leben und körperliche Unversehrtheit, in: HdStR VI, 2. A. 2001, § 128, Rn. 62; Schwabe, Probleme der Grundrechtsdogmatik, 1977, S. 51, Fn. 57; Würtenberger/Heckmann/Riggert, Polizeirecht in Baden-Württemberg, 5. A. 2002, Rn. 402. 
ser Ansicht folgen, jedoch nicht den offensichtlichen Widerspruch zwischen ihrer Auffassung und der Rechtsprechung des Bundesverfassungsgerichts, das Art. 2 I GG gerade unter Zurückstellung von Wortlautargumenten seit jeher sehr weit auslegt. ${ }^{4}$ Für ein Verständnis der Vorschrift als allgemeines Freiheitsrecht spricht insbesondere Art. 1 I des Herrenchiemseer Entwurfs, der eine auch für das Staatsverständnis des Grundgesetzes anerkannte Aussage trifft: »Der Staat ist um des Menschen willen da, nicht der Mensch um des Staates willen. $\aleph^{5}$ Diese »Menschbezogenheit « ist auch der inhaltliche Grund für die weite Auslegung des Art. 2 I GG: Denn wenn der Staat für den Menschen da ist, so ist der Staat eben kein Wert an sich, sondern nur Mittel zum Zweck. Ihm kann daher auch keine Befugnis zur Freiheitsbeschränkung zukommen, für die er sich nicht rechtfertigen muss. Würde man Art. 2 I GG in einem engen Sinne verstehen, so gäbe es Bereiche menschlicher Freiheit, in die der Staat eingreifen könnte, ohne dass er sich dafür rechtfertigen müsste bzw. die Betroffenen sich dagegen wehren könnten. Das widerspricht aber den Grundgedanken des Art. 1 I des Herrenchiemseer Entwurfs und der Menschenwürde. Treffend formuliert Alexy: »[D]er ... Respekt vor den Entscheidungen und Lebensformen des Einzelnen ... verlangt, dass nicht ohne hinreichenden Grund eingegriffen wird.« ${ }^{6}$

Wendet man diesen Gedanken auf die hier interessierende Problematik an, so ergibt sich, dass der Selbstmord von Art. 2 I GG geschützt sein muss. Denn wenn dies nicht der Fall wäre, hätte der Staat das Recht, in die Freiheit des Einzelnen sich selbst zu töten, einzugreifen, ohne sich dafür rechtfertigen zu müssen. An die Rechtfertigung werden im Rahmen des Art. 2 I GG nur geringe Anforderungen gestellt: Der Staat muss darlegen, dass die Freiheitsbeschränkung geeignet und erforderlich zur Erreichung eines legitimerweise verfolgten Zwecks ist. Wenn der Staat also Selbstmorde verhindern will, so darf der Bürger erwarten, dass der Staat dafür einen legitimen Zweck anzugeben in der Lage ist und dass das staatliche Eingreifen zur Förderung dieses Zwecks geeignet und erforderlich ist. Findet sich ein solcher legitimer Zweck nicht, so hat der Staat kein Recht, jemanden am Selbstmord zu hindern. Eben dies besagt Art. 2 I GG in seiner weiten Auslegung. Daher geht das erste Argument gegen ein Recht auf Selbstmord, das auf den Wortlaut des Art. 2 I GG abstellt, fehl, denn es verkennt, dass der materielle Grund für die weite Auslegung des Art. 2 I GG auch auf den Fall des Selbstmordes anwendbar ist.

Der zweite Einwand lautet, dass die »positive Wertentscheidung für das Leben«, die aus Art. 2 II GG folge, einen Schutz des Selbstmordes untersage, ${ }^{7}$ weshalb es »gestattet« sei, dass der Staat dem Grundrechtsinhaber die Verfügungsgewalt über das Leben

4 BVerfGE 6, 32 (36 ff.); 80, 137 (154).

5 Zur Verankerung dieser Aussage in der Menschenwürdegarantie des Grundgesetzes vgl. Stein/Frank, Staatsrecht, 18. A. 2002, S. 247.

6 Alexy, Theorie der Grundrechte, 1986, S. 325, Fn. 67. Vgl. auch Hochhuth, Lückenloser Freiheitsschutz und die Widersprüche des Art. 2 Abs. 1 GG, in: JZ 2002, 743 (748), mit einer ähnlichen Begründung.

7 BayObLG, NJW 1989, 1815 (1816); Götz (Fn. 3), Rn. 109; Würtenberger, in: Achterberg/ Püttner/Würtenberger, Besonderes Verwaltungsrecht II, 2. A. 2000, § 21, Rn. 182; Würtenberger/Heckmann/Riggert (Fn. 3), Rn. 402. 
abspreche ${ }^{8}$ dieses sei als Basis und Ausdruck menschlicher Existenz jeder Verfügung entzogen. ${ }^{9}$ Besonders stichhaltige Argumente sind das nicht. Warum ist etwas jeder Verfügung entzogen, nur weil es Basis und Ausdruck menschlicher Existenz ist? In faktischer Hinsicht ist das eigene Leben der Verfügung sicherlich nicht entzogen (fast) jeder hat die Möglichkeit, es zu beenden. Gemeint sein muss wohl entweder, dass der Einzelne nicht über sein eigenes Leben verfügen sollte, oder dass das Recht die faktische Verfügbarkeit des eigenen Lebens nicht durch einen grundrechtlichen Schutz aufgreifen und anerkennen sollte - in beiden Fällen fehlt jedoch weiterhin eine Begründung. Inwieweit Art. 2 II GG eine positive Wertentscheidung über das Leben enthält, wird weiter unten untersucht werden. Selbst wenn man aber eine solche positive Wertentscheidung annehmen würde, so würde das eher dazu führen, dass ein Selbstmord unterbunden werden kann, weil der damit verbundene Eingriff in das allgemeine Freiheitsrecht aufgrund der objektiven Wertentscheidung des Art. 2 II GG gerechtfertigt wäre. Art. 2 I GG stellt die Regel auf, dass jede Beschränkung subjektiver Freiheit durch einen sachlichen Grund gerechtfertigt werden muss; naheliegenderweise wird man das auf der Ebene der Rechtfertigung untersuchen.

Der dritte Einwand schließlich lautet, dass der Selbstmord selten oder nie Ausdruck freier Selbstbestimmung sei und deshalb kein grundrechtlich geschütztes Recht auf Selbstmord bestehen könne. ${ }^{10}$ Auch dieses Argument wird weiter unten ausführlich behandelt werden. Wiederum aber gibt es nichts dafür her, den Selbstmord vom Schutzbereich des allgemeinen Freiheitsrechts auszunehmen. Denn dann dürfte sich auch ein Betrunkener oder Geisteskranker nicht auf das Freiheitsrecht berufen, sondern wäre schutzlos, weil seine Handlungen ebenfalls nicht selbstbestimmt sind. Diese Konsequenz wird aber nicht gezogen; ${ }^{11}$ daraus folgt, dass auch dieses Argument richtigerweise allenfalls auf Rechtfertigungsebene eine Rolle spielen kann.

\section{Schutz des Selbstmordes durch das allgemeine Persönlichkeitsrecht}

Merkwürdigerweise wurde die Frage, ob der Selbstmord auch durch das allgemeine Persönlichkeitsrecht geschützt ist, bisher vernachlässigt. Der Grund dafür mag in der Auffassung liegen, das allgemeine Freiheitsrecht schütze Handlungen, während das allgemeine Persönlichkeitsrecht nicht das »Tun«, sondern das »Sein« des Menschen schütze. ${ }^{12}$ Auf Basis dieses Ansatzes wäre der Selbstmord als »Tun« dann der Handlungsfreiheit zuzuordnen.

8 Dürig, in: Maunz/Dürig, GG, Vorauflage, Art. 2 II, Rn. 12.

9 Lorenz (Fn. 3), Rn. 62.

10 Götz (Fn. 3), Rn. 109; Martens, Der Schutz des Einzelnen im Polizei- und Ordnungsrecht, in: DÖV 1976, 457 (459).

11 Vgl. dazu nur Rüfner, Grundrechtsträger, in: HdStR V, 2. A. 2000, § 116, Rn. 27.

12 Alexy (Fn. 6), S. 333; Dreier, in: Dreier (Fn. 2), Art. 2 I, Rn. 23; Dürig, Die Menschenauffassung des Grundgesetzes, in: JR 1952, 259 (261); Murswiek (Fn. 2), Art. 2, Rn. 59; Pieroth/Schlink, Grundrechte, 19. A. 2003, Rn. 373; Schmitt Glaeser, Schutz der Privatsphäre, in: HdStR VI, 2. A. 2001, § 129, Rn. 19; Starck, in: v. Mangoldt/Klein/Starck (Hrsg.), GG, 4. A. 1999, Art. 2 I, Rn. 83. 
Zutreffenderweise erfasst das allgemeine Persönlichkeitsrecht aber nicht nur das »Sein« des Menschen, sondern teilweise auch das »Tun«. Denn das Persönlichkeitsrecht rechtfertigt seine Existenz daher, dass das allgemeine Freiheitsrecht des Art. 2 I GG in der vom Bundesverfassungsgericht favorisierten weiten Auslegung zu weit erscheint, um einen wirksamen Persönlichkeitsschutz zu gewährleisten: Zum einen erfasst es so unterschiedlich zu gewichtende Verhaltensweisen wie die sexuelle Selbstbestimmung auf der einen und Taubenfüttern auf der anderen Seite; zum anderen ist die weitgehende Einschränkbarkeit des Freiheitsrechts für besonders persönlichkeitsrelevante Bereiche unangemessen. Daher muss das Persönlichkeitsrecht all die Positionen mit einem verstärkten Schutz versehen, die für die Integrität des Einzelnen besonders wichtig sind. Dazu gehören aber auch Handlungen: Es kann für den Integritätsschutz unabdingbar sein, sich in bestimmter Weise verhalten zu können. Wie sich der Einzelne in Bezug auf Fragen der Sexualität, der Familienplanung, der Berufswahl, um nur einige Beispiele anzudeuten, verhält, hat in der Regel größte Auswirkungen auf seine seelische Integrität. Wenn demgegenüber geäußert wird, das Persönlichkeitsrecht schütze »vor dem unbefugten Eindringen in einen gewissermaßen bereits materialisierten oder gefestigten Status $«{ }^{13}$ so scheint dem ein Persönlichkeitsverständnis zugrunde zu liegen, wonach sich ein bestimmter, einmal erreichter »Stand « oder »Status « der Persönlichkeitsentwicklung gleichsam konservieren und auf diese Weise »sichern« ließe. Das Persönlichkeitsrecht darf sich aber nicht damit begnügen, das zu schützen, was bereits geschaffen wurde. Erforderlich für einen umfassenden Persönlichkeitsschutz ist vielmehr zusätzlich der Schutz vor staatlicher Beeinträchtigung der weiteren, auf dem Erlangten aufbauenden Persönlichkeitsentfaltung. Persönlichkeitsschutz als Integritätsschutz ist daher immer auch Aktivitätsschutz. ${ }^{14}$

Begreift man das Persönlichkeitsrecht dann als ein Recht, sich in ethischen Fragen von hoher Präferenz, also solchen Fragen, die sich dadurch auszeichnen, dass sie das Selbstverständnis des Einzelnen berühren, autonom entscheiden zu können, oder plakativer - als ein Recht auf Selbstverwirklichung, ${ }^{15}$ so liegt eine Einbeziehung des Selbstmordes nahe. Zwar mag man der Ansicht sein, ein Leben sei nur dann ein gut gelebtes, wenn es mit einem natürlichen Tod endet. Jedoch wird auch die Entscheidung, sich selbst zu töten, Ausdruck einer ethischen Position sein. Beispielsweise kann der Betreffende der Auffassung sein, zu einem selbstbestimmt gelebten Leben gehöre auch ein selbstbestimmtes Ende, oder ein Schwerstkranker kann davon ausgehen, ein Ende unter von ihm als sinnlos und entwürdigend empfundenen Schmerzen entspreche weniger seinem Lebensplan als der Freitod. In solchen Fällen liegt es auf der Hand, dass sich in der Entscheidung für den Selbstmord eine ethische Position von hoher Präferenz ausdrückt.

Aber kann dies wirklich für jeden Selbstmord angenommen werden? Wie ist es mit demjenigen, der einfach aus Verzweiflung über seine Schulden oder darüber, dass ihn seine Freundin verlassen hat, Selbstmord begeht? Bei genauer Betrachtung zeigt sich,

13 Dreier (Fn. 12), Art. 2 I, Rn. 23.

14 Vgl. dazu ausführlich Möller, Paternalismus und Persönlichkeitsrecht, 2005, S. 58 ff., 63 ff.

15 Möller (Fn. 14), S. 63 ff., 73 ff. 
dass auch in diesen Fällen das allgemeine Persönlichkeitsrecht eingreift. Denn der Grund für die Selbsttötung liegt auch in diesen Fällen darin, dass der Betroffene davon ausgeht, dass der Tod besser sei, als das Leben unter den gegebenen Umständen weiterleben zu müssen; insofern manifestiert sich auch in solchen Taten eine individuelle Entscheidung über das gute, oder vielleicht: am wenigsten verfehlte Leben. Man mag das als unvernünftig ansehen oder vielleicht die Freiwilligkeit und Ernsthaftigkeit eines unter solchen Umständen gefassten Entschlusses anzweifeln. Wie sich solche Mängel auswirken, ist jedoch, wie dargelegt, keine Frage, die im Schutzbereich eines Grundrechts zu entscheiden ist, sondern auf Rechtfertigungsebene. Auf Schutzbereichsebene dagegen ist der Selbstmord nicht nur vom Freiheitsrecht, sondern auch vom allgemeinen Persönlichkeitsrecht erfasst. ${ }^{16}$

\section{Schutz des Selbstmordes durch Art. 2 II GG?}

Teilweise wird auch vertreten, dass der Selbstmord nicht nur durch Art. 2 I GG, sondern auch durch Abs. 2, also das Recht auf Leben, geschützt sein soll. ${ }^{17}$ Das erscheint deshalb problematisch, weil Abs. 2 als Abwehrrecht gegen staatliche Eingriffe in das Leben gemeint ist und nicht eine Freiheit, zu leben oder es eben bleiben zu lassen, schützen soll. ${ }^{18}$ Die Meinung, die den Selbstmord unter Abs. 2 subsumieren will, hat jedoch insofern ein berechtigtes Anliegen, als ein Schutz des Selbstmordes nur durch das allgemeine Freiheitsrecht unzureichend wäre, da der Selbstmord dann auf einer Stufe mit Taubenfüttern oder Reiten im Walde geschützt wäre; die besondere Bedeutung einer Selbsttötung kann durch den Schutz des allgemeinen Freiheitsrechts nicht adäquat erfasst werden. Besser als Art. 2 II GG ist dafür jedoch das allgemeine Persönlichkeitsrecht geeignet, denn beim Selbstmord geht es, wie dargelegt, in erster Linie um die Umsetzung einer Entscheidung von hoher ethischer Präferenz. So kann man einen gegenüber dem allgemeinen Freiheitsrecht erhöhten Schutz des Selbstmordes erreichen, ohne die künstlich wirkende Konstruktion über das Recht auf Leben bemühen zu müssen.

\section{Die Grenzen des Rechts auf Selbstmord}

\section{Die objektive Dimension der Grundrechte}

Es wurde dargelegt, dass der grundrechtliche Schutz des Selbstmordes von einigen Autoren unter Hinweis auf die objektive Dimension der Grundrechte verneint wird, und dass dieses Argument zutreffenderweise nicht im Rahmen des Schutzbereichs sondern der Rechtfertigung zu erörtern ist. Die Untersuchung wird an dieser Stelle dadurch zu einer schwierigen Aufgabe, dass sich die Verfassung zu einer möglichen objektiven Dimension der Grundrechte nicht explizit äußert. Man kann allenfalls einige

16 Im Ergebnis ebenso Günzel, Das Recht auf Selbsttötung, seine Schranken und die strafrechtlichen Konsequenzen, 2000, S. 95 ff. Angedeutet bei Sachs (Fn. 2), B 2, Rn. 81.

17 Fink, Selbstbestimmung und Selbsttötung, 1992, S. 110; Pieroth/Schlink (Fn. 12), Rn. 392.

18 Schwabe, Der Schutz des Menschen vor sich selbst, in: JZ 1998, 66 (69). 
dünne Hinweise finden, die darauf hindeuten mögen, dass die Grundrechte nicht lediglich als subjektive Abwehrrechte gegen den Staat gedacht sind, sondern auch andere Wirkungsweisen haben. So fordert Art. 1 I 2 GG nicht nur die Achtung, sondern auch den Schutz der Menschenwürde durch die staatliche Gewalt. Abs. 3 stellt fest, dass die Grundrechte die drei Gewalten als unmittelbar geltendes Recht binden. Das wird teilweise als Hinweis auf die objektive Dimension der Grundrechte verstanden. ${ }^{19}$ Eine solide textliche Grundlage, die schon aus sich heraus eine extensive Interpretation der Grundrechte erforderlich macht, bietet die Berufung auf Art. 1 I und III GG aber nicht.

Das Bundesverfassungsgericht hat trotz allem schon früh neben der traditionellen abwehrrechtlichen Seite der Grundrechte eine weitere Grundrechtsdimension ausgemacht. In der Lüth-Entscheidung heißt es: »Ohne Zweifel sind die Grundrechte in erster Linie dazu bestimmt, die Freiheitssphäre des Einzelnen vor Eingriffen der öffentlichen Gewalt zu sichern; sie sind Abwehrrechte des Bürgers gegen den Staat ... Ebenso richtig ist aber, dass das Grundgesetz, das keine wertneutrale Ordnung sein will, in seinem Grundrechtsabschnitt auch eine objektive Wertordnung aufgerichtet hat. ${ }^{20}$ Auf Basis dieser isolierten Aussage könnte man zu dem Schluss kommen, dass die in den Grundrechten ausgedrückten Werte durchaus auch zur Einschränkung der Freiheit des Grundrechtsträgers selbst herangezogen werden könnten: Wenn beispielsweise Art. 2 II GG eine Anerkennung der Werte Leben und Gesundheit enthält, und diese Werte in alle Richtungen und objektiv wirken, dann ist auch derjenige von der Wertgeltung erfasst, der sie für sich ablehnt: Der Geltung von objektiven Werten kann eben keiner entrinnen. ${ }^{21}$ Allerdings fährt das Bundesverfassungsgericht an der zitierten Stelle fort und fügt hinzu: » ... und dass gerade hierin eine prinzipielle Verstärkung der Geltungskraft der Grundrechte zum Ausdruck kommt.« Das ändert die Sachlage erheblich: Wenn die (und man darf hinzufügen: vorrangig subjektivrechtliche) Geltungskraft der Grundrechte verstärkt werden soll, kann das nicht dadurch gelingen, dass der subjektiven Freiheit des Einzelnen ein objektiver Wert entgegengehalten und so eine Freiheitsbeschränkung legitimiert wird. In diesem Sinne äußert sich das Gericht auch im Mitbestimmungsurteil: »Nach ihrer Geschichte und ihrem heutigen Inhalt sind sie in erster Linie individuelle Rechte, Menschen- und Bürgerrechte, die den Schutz konkreter, besonders gefährdeter Bereiche menschlicher Freiheit zum Gegenstand haben. Die Funktion der Grundrechte als objektiver Prinzipien besteht in der prinzipiellen Verstärkung ihrer Geltungskraft, hat jedoch ihre Wurzel in dieser primären Bedeutung. Sie lässt sich deshalb nicht von dem eigentlichen Kern lösen und zu einem Gefüge objektiver Normen verselbständigen, in dem der ursprüngliche und bleibende Sinn der Grundrechte zurücktritt. $\ll^{22}$

In juristisch-dogmatischer Sicht scheint diese Auffassung überzeugend, wenn nicht sogar zwingend. Gerade am Beispiel des Selbstmordes lässt sich zudem treffend eine

19 Pieroth/Schlink (Fn. 12), Rn. 77. Kritisch zu dieser Interpretation des Art. 1 III GG aber zu Recht Böckenförde, Staat, Verfassung, Demokratie, 1991, S. $160 \mathrm{f}$.

20 BVerfGE 7, 198 (204 f.).

21 Vgl. Böckenförde, Recht, Staat, Freiheit, 1991, S. 73 f.

22 BVerfGE 50, 290 (337). 
weitere Schwäche des Konzepts der objektiven Werte darstellen. Wer dafür streitet, dass aufgrund der objektiven Dimension der Grundrechte der Selbstmord eingeschränkt werden kann, der hat keine Möglichkeit, zwischen verschiedenen Fällen des Selbstmordes zu differenzieren. Wenn Leben ein objektiver »Wert « ist, dann »gilt « dieser Wert ebenso im Fall des von seiner Freundin verlassenen Studenten wie in dem des unerträglich leidenden Krebskranken im Endstadium. Wer dazu geneigt ist, zumindest im letzteren Fall den Selbstmord zuzulassen, der bekommt Probleme, wenn er ansonsten die Theorie der objektiven Werte bejaht. Es gibt keinen sachlichen Differenzierungsgrund, mit dem man erklären könnte, warum dem liebeskranken Studenten der Selbstmord wegen der Geltung des Wertes »Leben« versagt werden soll, der Krebskranke dagegen möglicherweise das Recht haben soll, sein Leben zu beenden. Denn gerade aus der Eigenschaft als Wert folgt die Geltungskraft unabhängig vom Einzelfall. ${ }^{23}$

\section{Willensmängel}

\section{a) Geisteskrankheit oder Schuldunfähigkeit}

In der Grundrechtsdogmatik ist anerkannt, dass die Willensbildung bei Geisteskranken und Minderjährigen solche Defizite aufweisen kann, dass ein Einschreiten gerechtfertigt ist. ${ }^{24}$ Es bestünde also Hoffnung, die Rettung von Selbstmördern ohne systematische Brüche rechtfertigen zu können, wenn dargelegt werden könnte, dass sich diese regelmäßig in einem Zustand der Unzurechnungsfähigkeit befinden. Geilen hat in der Literatur viel Unterstützung für seinen Versuch erhalten, die Ergebnisse der Suizidforschung in die rechtliche Bewertung des Selbstmordes einfließen zu lassen. Er beruft sich insbesondere auf Ringel, der zu dem Ergebnis gekommen war, dass es das Phänomen des normalpsychologischen Bilanzselbstmordes gar nicht gebe und fast jeder Selbstmord als Endpunkt einer Krankheit oder einer krankhaften Entwicklung angesehen werden müsse. ${ }^{25}$

Die Ergebnisse Ringels werden in der modernen Suizidologie jedoch durchaus kritisch gesehen; Fink bemerkt, dass eine stärkere Zurückhaltung mit dem Begriff

23 Vgl. Böckenförde (Fn. 21), S. 78 (»abstrakt und allgemein«, »auf Totalität der Geltung gerichtet«), 88 (»abstrakte und universale Geltung«). Daher ist es verwunderlich, dass in der Literatur auf einmal ohne nähere Begründung Ausnahmen von der objektiven Geltung der Grundrechte zugelassen werden, wenn das eigene Rechtsempfinden dies gerade nahe legt. Die Ergebnisse, die dabei herauskommen, lassen so manchen Schluss auf die Weltanschauungen des jeweiligen Autors zu, haben aber wenig mit einer konsequenten und widerspruchsfreien Anwendung des Konzepts der objektiven Werte gemein. Ein Beispiel: Dürig zufolge soll der »Verzicht auf das eigene Leben« auf einmal doch wieder »rechtsbeachtlich« sein, wenn er selbstlos zur Rettung anderer Leben erfolgt, wenn etwa die Mutter unter Lebensgefahr einen medizinisch indizierten Schwangerschaftsabbruch ablehnt, um ihr Kind zu retten (Dürig [Fn. 8], Art. 2 II, Rn. 12).

24 Hillgruber, Der Schutz des Menschen vor sich selbst, 1992, S. 121.

25 Vgl. Geilen, Suizid und Mitverantwortung, in: JZ 1974, 145 (152) m.w.N. Zustimmend BayVerfGH, NJW 1989, 1790 (1791); Dölling, Suizid und unterlassene Hilfeleistung, in: NJW 1986, 1011 (1014); Knemeyer, Der Schutz der Allgemeinheit und der individuellen Rechte durch die polizei- und ordnungsrechtlichen Handlungsvollmachten der Exekutive, in: VVDStRL 35, 221 (254 f.); Martens (Fn. 10), S. 459. 
»krankhaft« zu beobachten ist. Zum einen spreche die neuere Depressionsforschung den meisten ihrer Erscheinungsformen eine solche Qualität ab, so dass im Wesentlichen nur noch die Fälle manischer Depression gerade auch mit Blick auf die rechtliche Kategorienbildung Bedeutung gewinnen sollten. Zum anderen werde der Nachweis einer selbsttötungsspezifischen Verengung der Handlungsfreiheit aus erkenntnistheoretischen Gründen angezweifelt. Es ergebe sich das Bild einer multifaktoriellen, äußerst komplexen Problemlage, die eine Monokausalität im Sinne Ringels als nicht mehr diskutierbar erscheinen lasse und auch dem Gedanken eigenverantwortlicher Handlungsgestaltung Raum geben müsse. ${ }^{26}$

Mit dem in der Grundrechtsdogmatik verwendeten Begriff der »Geisteskrankheit « kommt man daher zumeist nicht weiter, denn insbesondere bei dem sog. Appellselbstmord liegt häufig keine Krankheit im medizinischen Sinn vor. Auch wenn man die Freiverantwortlichkeit in Anlehnung an $\S 20$ StGB bestimmt - was nahe liegen könnte, da $§ 20$ StGB eine Ausprägung des verfassungsrechtlichen Schuldprinzips ist $^{27}$-, so führt dies dazu, dass viele Selbstmorde als freiverantwortlich klassifiziert werden müssen, denn es mögen zwar gewisse Einschränkungen der Willensbildung vorliegen; diese erreichen jedoch nicht den Grad der Schuldunfähigkeit. ${ }^{28}$

\section{b) Geäußerter versus »wahrer« Wille}

Soweit ein prinzipielles, durch die Wertentscheidung des Art. 2 II GG auch nicht weiter einschränkbares Recht auf Selbstmord in der staats- oder polizeirechtlichen Literatur anerkannt wird, stellen die Autoren zumeist auf eine Unterscheidung zwischen einem Appellselbstmord, bei dem der Betreffende seine Umgebung lediglich auf seine verzweifelte Lage aufmerksam machen will, und einem »rationalen« Bilanzselbstmord ab. ${ }^{29}$ Hillgruber folgert sodann kühn: »Diese Unterscheidung ist verfassungsrechtlich insofern bedeutsam, als derjenige, der eine Selbsttötungshandlung mit Appellfunktion vornimmt, zwar den Tod als Folge seines bewusst lebensgefährdenden Verhaltens voraussieht und in Kauf nimmt, aber nicht als Handlungsziel anstrebt, d.h. nicht unbedingt zum Freitod entschlossen ist, vielmehr in Wahrheit ${ }^{30}$ gerettet werden will. In diesem Fall stellt sich daher polizeiliches Eingreifen gar nicht als rechtfertigungsbedürftiger Eingriff dar, weil es bei der Einleitung lebensrettender Maßnahmen keinen entgegenstehenden Willen des davon Betroffenen zu überwinden gilt. « ${ }^{31}$

Die Sache scheint also ganz einfach zu sein. Im ersten Schritt wird eine Unterscheidung vorgenommen zwischen dem geäußerten und dem »wahren« Willen des Betrof-

26 Fink (Fn. 17), S. 159 f., m.w.N.

27 Jähnke, in: Leipziger Kommentar zum StGB, 11. A. 1992 ff., § 20, Rn. 3.

28 Dölling (Fn. 25), S. 1014; Pohlmeier, Freiverantwortlichkeit des Suizids aus medizinischpsychologischer Sicht, in: Pohlmeier/Schöch/Venzlaff (Hrsg.), Suizid zwischen Medizin und Recht, 1996, S. 33 (37).

29 Hillgruber (Fn. 24), S. 87; Dölling (Fn. 25), S. 1015; von Münch, Grundrechtsschutz gegen sich selbst?, in: FS Ipsen, 1977, S. 113 (122); ähnlich (Erfordernis des »völlig klaren Bewusstseins «) Murswiek (Fn. 2), Art. 2, Rn. 211.

30 Hervorhebung d. Verf.

31 Hillgruber (Fn. 24), S. 87 f. 
fenen, und im zweiten Schritt wird der »wahre« Wille, wenig überraschend schon aufgrund der Terminologie, zum maßgeblichen erklärt. Die Figur des »wahren« Willens ist jedoch, milde gesprochen, problematisch. Wenn sich jemand - und das soll im Moment unterstellt werden: bei klarem Bewusstsein - die Pulsadern aufschneidet oder eine Überdosis Medikamente einnimmt, so scheint es nicht weit hergeholt, davon auszugehen, dass der Betroffene den eigenen Tod anstrebt. Man kann erwachsenen Menschen nicht ohne weitere Erörterung einfach unterstellen, dass bei derart gravierenden und gefährlichen Eingriffen in die eigene körperliche Integrität »alles nicht so gemeint « sei. Auch Geilen gibt zu, dass es sich keinesfalls in der Mehrzahl der Fälle um »theatralische Selbstmorddemonstrationen « handele, sondern vielmehr ein subjektiv durchaus ernsthafter Selbsttötungswille mit einer unterschwellig ambivalenten und deshalb die Ausführung bremsenden Handlung zusammentreffen könne. ${ }^{32}$

Wenn also ein Selbstmörder gerettet wird, so liegt darin entgegen Hillgruber sehr wohl eine Handlung, die gegen seinen subjektiven und sich in der Tat manifestierenden Willen vorgenommen wird. Man kann die Situation noch verschärfen und sich den Fall vorstellen, dass der Lebensmüde die Hilfe der Retter wortreich ablehnt und darum bittet, in Ruhe gelassen zu werden. Wie weit soll die Überspielung des geäußerten Willens durch den »wahren « Willen möglich sein? Wenn man auch in Fällen, in denen jemand bei vollem Bewusstsein darum bittet, in Ruhe gelassen zu werden, einen aufgezwungenen Schutz damit rechtfertigen will, man setze sich »in Wahrheit« gar nicht über den Willen des Betreffenden hinweg, dann kann man auf die Idee der Selbstbestimmung auch gleich verzichten - alles, was dann nicht ins gewünschte Bild passt, wird übergangen durch eine Berufung auf den »wahren « Willen. Man könnte auf diese Weise beispielsweise das ganze Medizinrecht neu gestalten: Wer eine Operation ablehnt, obwohl sie medizinisch indiziert ist, der kennt seinen eigenen wahren Willen noch nicht! Eine lebenserhaltende Operation ist durchaus vergleichbar mit der Rettung eines Selbstmörders, da es in beiden Fällen um die Beseitigung einer lebensgefährdenden Situation geht. Die Figur des »wahren « Willens ist also problematisch. Sie kann möglicherweise zwar durchaus einen richtigen Kern haben: Allgemein gesprochen könnte man sagen, dass der Entscheidung des Lebensmüden so gravierende Mängel zugrunde liegen, dass ein Einschreiten auch gegen dessen erklärten Willen gerechtfertigt ist. Dann gälte es aber, Kriterien zu entwerfen, wann ein Überspielen des Willens eines geistig gesunden Erwachsenen zu rechtfertigen ist.

\section{c) Integritätswidriges Verhalten}

Damit kristallisiert sich der entscheidende Ansatzpunkt zur Beurteilung der Zulässigkeit der Rettung von Selbstmördern heraus: Die Frage ist, ob man einer Person, die sich nicht im Zustand der Unzurechnungsfähigkeit befindet, in paternalistischer Weise einen ihrem natürlichen Willen zuwider laufenden Schutz aufzwingen kann.

Die naheliegende liberale Position zu staatlichem Paternalismus ist, dass es vom Autonomierecht einer Person umfasst ist, sich freiwillig selbst zu gefährden oder ver-

32 Geilen (Fn. 25), S. 153. 
letzen. Am eindrucksvollsten haben diese Position John Stuart Mill und Joel Feinberg entwickelt und vertreten. ${ }^{33}$ Feinberg vergleicht das Autonomierecht des Einzelnen mit dem Souveränitätsrecht der Staaten. So wie ein Staat sein Souveränitätsrecht nicht verliere, nur weil er schlecht regiert werde, verliere auch das Individuum sein Autonomierecht nicht schon aus dem Grund, dass es nachteilige Entscheidungen für sich treffe. $^{34}$

Der Sache nach ist Feinbergs Ansatz auch in der Grundrechtsdogmatik anerkannt; er spiegelt sich in der prominent von Hillgruber vertretenen Position wider, dass staatlicher Paternalismus nur dann zulässig sei, wenn er sich auf Minderjährige oder Geisteskranke beziehe. ${ }^{35}$ Diese Auffassung kommt jedoch regelmäßig bei der Selbstmordproblematik in Schwierigkeiten, wenn sie die Zulässigkeit der Rettung von Selbstmördern bejaht. Denn wie dargelegt, kann eine Unfreiwilligkeit oder gar Geisteskrankheit bei Selbstmördern keinesfalls regelmäßig unterstellt werden. Dann müsste man folgerichtig die Zulässigkeit der Rettung von Selbstmördern verneinen, oder aber sich von dem zunächst einleuchtend erscheinenden Prinzip verabschieden, dass aufgedrängter Schutz nur bei Geisteskranken oder Minderjährigen zulässig sein soll.

Der Verfasser hat in seiner Dissertation »Paternalismus und Persönlichkeitsrecht « dargelegt, dass staatlicher Paternalismus unter gewissen Umständen auch bei geistig gesunden Erwachsenen gerechtfertigt werden kann. Ausgangspunkt ist die Erkenntnis, dass staatlicher Paternalismus in das allgemeine Persönlichkeitsrecht des Betroffenen eingreift, denn dieses schützt die Autonomie des Einzelnen, ohne staatliche Bevormundung darüber zu entscheiden, welchen Gefahren er sich aussetzen will. ${ }^{36}$ Dieser Eingriff kann jedoch gerechtfertigt sein, wenn es dem Staat um den Schutz der Integrität des Betroffenen geht. Das Konzept des Integritätsschutzes greift einen von Kleinig entwickelten Gedanken auf, demzufolge zu unterscheiden ist zwischen einem Schutz, der dem Betroffenen äußere, von ihm abgelehnte Werte aufzwingt, und einem Schutz, der dem Betroffenen dabei hilft, seine eigenen Werte zu verwirklichen. ${ }^{37}$ Das beste Beispiel für einen Fall, in dem Paternalismus erlaubt ist, ist die Sitzgurtpflicht. Obwohl derjenige, der seinen Sitzgurt nicht benutzt, regelmäßig freiwillig handelt, besteht ein Konflikt zwischen dem Fahren ohne Gurt und den eigenen Werten der Person, wenn der Betreffende keine Verletzung anstrebt, in dem Fahren ohne Gurt kein Element der Selbstverwirklichung (etwa durch einen besonderen »Kick«) liegt und der Betroffene letztlich nur aus Nachlässigkeit den Gurt nicht benutzt. Daher darf der

33 Vgl. Mills berühmtes harm principle: »Dies Prinzip lautet: dass der einzige Grund, aus dem die Menschheit, einzeln oder vereint, sich in die Handlungsfreiheit eines ihrer Mitglieder einzumischen befugt ist, der ist: sich selbst zu schützen. Dass der einzige Zweck, um dessentwillen man Zwang gegen den Willen eines Mitglieds der zivilisierten Gemeinschaft rechtmäßig ausüben darf, der ist: die Schädigung anderer zu verhüten. Das eigene Wohl, sei es das physische oder das moralische, ist keine genügende Rechtfertigung ... Über sich selbst, über seinen eigenen Körper und Geist ist der Einzelne souveräner Herrscher.« (Mill, Über die Freiheit, zitiert nach der Reclam-Ausgabe 1974, S. 16 f.).

34 Feinberg, Harm to Self, 1986, S. 52 ff.

35 Hillgruber (Fn. 24), S. 121.

36 Vgl. ausführlich Möller (Fn. 14), S. 95 ff.

37 Kleinig, Paternalism, 1984, S. 67 ff. 
Staat zum Schutz der eigenen Werte des Betroffenen eingreifen und diesem die Benutzung des Sitzgurtes vorschreiben.

Der dogmatische Grund für die Zulässigkeit des Integritätsschutzes liegt gerade in dem Sinn und Zweck des allgemeinen Persönlichkeitsrechts. Kern des Persönlichkeitsrechts ist der Schutz der Selbstverwirklichung des Einzelnen; dieser soll die in ihm angelegten Begabungen realisieren können. Dies wird aber nicht nur dadurch erreicht, dass man jede freiwillige Entscheidung des Einzelnen akzeptiert, sondern auch dadurch, dass man in den Fällen korrigierend eingreift, in denen der Einzelne seine Selbstverwirklichung durch unbedachte (aber gleichwohl freiwillige) Handlungen torpediert. Die begrenzte Zulässigkeit von Paternalismus folgt also aus der dadurch geförderten und von der Gewährleistung des Art. 2 I i.V.m. 1 I GG angestrebten Selbstverwirklichung. ${ }^{38}$

Angewendet auf die Selbstmordproblematik bedeutet dies: Der typische Fall, in dem die Berufung auf die Integrität des Betroffenen Paternalismus legitimieren kann, ist, dass der Einzelne seine Lebenspläne durch Unbedachtheiten torpediert. In vielen Fällen trifft dieser Gedanke auch auf den Selbstmord zu: Es handelt sich oft um »Kurzschlussreaktionen«, beispielsweise wenn sich ein junger Mann in den Kopf schießt, nachdem ihn seine Freundin verlassen hat. ${ }^{39}$ Hier überwiegen die Impulsivität und Unreflektiertheit der Beschlussfassung. ${ }^{40}$ In diesen Fällen spricht das Integritätsargument eindeutig für eine Rettung des Betroffenen.

Häufig ist die Sachlage jedoch eine andere: Gerade ein Appellselbstmord wird dann nicht aus Unbedachtheit ausgeführt, sondern eher aus Verzweiflung, und der Entschluss zur Tat kann möglicherweise schon lange gereift sein - man denke an vereinsamte alte Menschen, die aus Verzweiflung beschließen, ihrem Leben ein Ende zu setzen. ${ }^{41}$ Zudem handelt es sich dann viel deutlicher als in den Kurzschlussfällen gerade nicht um die Torpedierung, sondern um die Umsetzung eines »Lebensplanes «. Dies mag in Bezug auf den Selbstmord merkwürdig klingen. Jedoch liegt in der Selbsttötung auch die Aussage, dass das eigene Leben jetzt zu Ende gehen solle; insofern kann man von einem Lebensplan sprechen.

Aus diesen beiden Gründen liegt in einem Appellselbstmord häufig zumindest nicht der klassische Fall eines Paternalismus legitimierenden Verhaltens. Wenn man einen Appellselbstmord also als integritätswidrig ansehen möchte, muss man mehr in die Tiefe der Persönlichkeit des Suizidenten gehen. Es ist dann nicht ausreichend, die konkrete Handlung als widersprüchlich in Bezug auf die eigenen Lebenspläne anzusehen, wie dies beim Autofahren ohne Gurt möglich ist. Vielmehr ist zu untersuchen, ob der Lebensplan selbst, also die Überzeugung, das Leben solle beendet werden, in Widerspruch zu noch tieferen Überzeugungen und Ansichten des Betroffenen steht. Wenn das Weltbild des Suizidenten so ist, dass er unter veränderten Rahmenbedingungen weiterleben wollen würde, dann entspricht das angestrebte Ziel - der Tod - nur dann

38 Vgl. ausführlich Möller (Fn. 14), S. 181 ff.

39 Dölling (Fn. 25), S. 1014.

40 Geilen (Fn. 25), S. 152 m.w.N.

41 Dölling (Fn. 25), S. 1014. 
seinen Prioritäten, wenn keine Aussicht besteht, dass sich die Rahmenbedingungen ändern. Mit anderen Worten: Wenn der Staat sich nicht nur dafür einsetzen würde, dass das Leben des Betroffenen gerettet wird, sondern auch dafür Sorge tragen würde, dass der Betroffene es nach der Rettung wieder als lebenswert empfindet, dann wäre das mit der Tat verfolgte Ziel, nämlich sich selbst zu töten, auch nach den Maßstäben des Betroffenen selbst gar nicht erstrebenswert. Nach seinen eigenen Maßstäben wäre dann eine Rettung dem Tod vorzuziehen.

Wenn es heißt, dass der Betroffene unter anderen »Umständen« leben wolle und dass der Staat sich darum kümmern solle, dass der Suizident das Leben wieder als lebenswert empfindet, dann darf dies nicht missverstanden werden: Es geht hier nicht um eine Gesellschaftskritik o.ä. Erforderlich ist vielmehr in erster Linie eine entsprechende medizinische oder psychologische Betreuung. Das Therapieziel besteht darin, dem Patienten wieder zu einer nichtsuizidalen psychischen Verfassung zu verhelfen und ihm konstruktivere Weisen der Stressbewältigung und der Problemlösung zu vermitteln. ${ }^{42}$ Es wäre jedoch nicht nur zynisch, sondern auch eine Verletzung der Integrität des Betroffenen, einen alten Menschen, der an seiner Einsamkeit leidet und sich deshalb das Leben nehmen will, zunächst zu retten und dann ohne weitere Hilfestellung wieder seiner Einsamkeit zu überlassen. Der Integritätsansatz macht also die Legitimität der Rettung auch von den begleitenden Maßnahmen abhängig. ${ }^{43}$ In der Praxis wurde die Beobachtung gemacht, dass viele Menschen über ihre Rettung Dankbarkeit empfinden und in der Regel gute Aussichten bestehen, den Suizidenten endgültig für das Leben zurückzugewinnen. ${ }^{44}$ Das spricht dafür, dass in vielen Fällen geeignete Maßnahmen ergriffen worden sind, auch wenn in der psychologischen Literatur teilweise eine zu hohe Quote von Fällen, in denen gar keine fachgerechte Betreuung stattfindet, beklagt wird. ${ }^{45}$

Das Integritätsargument spricht also in Fällen des Appellsuizids für eine Rettung des Betroffenen. In Anbetracht der Größe des drohenden Schadens - dem Verlust des Lebens - erscheint es auch gerechtfertigt, die Befugnis des Betroffenen, über sein Leben freiwillig zu verfügen, in diesen Fällen einzuschränken. Als Indiz für die Richtigkeit dieser Abwägung kann auch die Beobachtung herangezogen werden, dass die meisten Geretteten für die Rettung dankbar sind und keinen erneuten Suizidversuch unternehmen. Das deutet darauf hin, dass sie selbst - wenn auch im Nachhinein - anerkennen, dass der paternalistische Eingriff gerechtfertigt war und ihren eigenen Interessen in legitimer Weise diente.

In keinem Fall kann dagegen eine Rettung von Suizidenten gerechtfertigt werden, bei denen eindeutig ein Bilanzselbstmord vorliegt. ${ }^{46}$ Dies wird beispielsweise bei Schwerkranken anzunehmen sein, für die das Leben eine Qual ohne Aussicht auf Bes-

42 Comer, Klinische Psychologie, 1995, S. 387 f.

43 So auch Kleinig (Fn. 37), S. 103.

44 Dölling (Fn. 25), S. 1014 m.w.N.

45 Comer (Fn. 42), S. 387, zitiert aus Quellen, denen zufolge in einem Helsinkier Allgemeinkrankenhaus $46 \%$ der wegen eines Suizidversuchs behandelten Personen keine psychologische Beratung erhielten.

46 So auch Dölling (Fn. 25), S. 1015. 
serung ist. Wenn sie zu dem Schluss kommen, dass der Tod die bessere Alternative zu einem Leben unter den gegebenen, nicht änderbaren Umständen sei, so erlaubt der Integritätsansatz kein Eingreifen. ${ }^{47}$

Gelegentlich hört man in der Diskussion auch den Hinweis, für ein Eingreifen des Staates spreche auch, dass dem Geretteten stets die Möglichkeit verbleibe, seinen Versuch zu wiederholen; niemand könne letztlich an einem solchen Schritt gehindert werden. ${ }^{48}$ Bei diesem Argument ist zu differenzieren: Zum einen gibt es die Fälle, in denen der Betroffene seinen Selbstmordversuch im Einklang mit seinen eigenen Werten durchführt. Wo eindeutig ein solcher Fall vorliegt, kann eine Rettung nicht damit begründet werden, der Betroffene könne ja hinterher erneut versuchen, sich das Leben zu nehmen - eine solche Argumentation wäre geradezu zynisch. Bei Appellselbstmorden dagegen ist anzustreben, dass der Suizident später nicht noch einmal versucht, sich das Leben zu nehmen. Gewicht hat das Argument daher nur, wenn unklar ist, ob ein Appell- oder ein Bilanzselbstmord vorliegt. Dann spricht für die Rettung zum einen die Möglichkeit, dass es sich um einen Appellselbstmord handelt, so dass das Einschreiten ohnehin gerechtfertigt wäre. Zum anderen liegt für den Fall, dass es sich um einen Bilanzselbstmord handelt, in der Rettung auch deshalb keine unzumutbare Belastung des Betroffenen, weil er einen erneuten Selbstmordversuch unternehmen kann. Dadurch muss er zwar durch die erneuten psychischen Belastungen eines Selbstmordversuches gehen, hat jedoch immerhin die tatsächliche Möglichkeit, seinen Plan umzusetzen. ${ }^{49}$

\section{Ergebnis}

Der Verfasser hofft, mit der hier vorgestellten Analyse der Selbstmordproblematik zweierlei erreicht zu haben. Erstens wurden die teilweise recht undifferenziert vorgetragenen Argumente für oder gegen einen grundrechtlichen Schutz des Selbstmordes systematisiert und den jeweils einschlägigen dogmatischen Ebenen zugeordnet. Zweitens wurden die inhaltlichen Fragen erörtert. Die Einwände, dass der Selbstmord nicht geschützt sei, weil er nicht der Entwicklung, sondern der Zerstörung der Persönlichkeit diene und zudem die positive Wertentscheidung zugunsten des Lebens einen Schutz untersage, wurden mit Argumenten zurückgewiesen, die heute wohl in hohem Maße konsensfähig sein dürften. ${ }^{50}$ Mit der grundrechtlichen Erfassung des Selbstmordes durch das allgemeine Persönlichkeitsrecht des Art. 2 I i.V.m. 1 I GG wurde ein bisher weitgehend unbeachteter Aspekt in die Debatte eingebracht. Des weiteren wurde die ganz überwiegend vertretene Auffassung, Appellselbstmörder handelten nicht freiwillig bzw. ihr wahrer Wille sei nicht auf Selbsttötung gerichtet, verworfen.

47 Ganz im Gegenteil: Die Sorge um die Integrität des Betroffenen legt in diesem Fall nahe, ihm bei der Umsetzung seines Suizids sogar zu helfen, wenn er es allein nicht mehr ausführen kann. Dabei entstehen dann jedoch neue Probleme, die im Rahmen der Diskussion um den $§ 216$ StGB diskutiert werden, etwa die Frage, wie ein Missbrauch verhindert werden kann. Darauf ist hier nicht einzugehen.

48 Götz (Fn. 3), Rn. 109.

49 In diesem Sinne Dölling (Fn. 25), S. 1015.

50 Ähnlich Deger (Fn. 2), 1230. 
Die einzige dogmatisch saubere Möglichkeit, in vielen Fällen die Zulässigkeit der Rettung von Selbstmördern zu begründen, liegt darin, den Schutz der Integrität der Betroffenen, also der von ihnen selbst bejahten Werte, für zulässig zu erklären, und auf dieser Basis die Rechtfertigungsmöglichkeiten auszuloten. Der sich auf diese Weise ergebende Lösungsansatz hat den Vorzug, die intuitiv vielen einleuchtende Zulässigkeit der Rettung von Selbstmördern theoretisch zu fundieren, ohne dabei auf die Widersprüche der Konzeption der h.M. zurückgreifen zu müssen. Zugleich lässt er Raum für notwendige Differenzierungen und gibt der Praxis, insbesondere was die begleitenden Maßnahmen einer Rettung angeht, wichtige Leitlinien vor. 